\title{
Asset Pricing and Ambiguity: Empirical Evidence ${ }^{\dagger}$
}

\author{
Menachem Brenner ${ }^{1}$ and Yehuda Izhakian ${ }^{2}$
}

This Version:

December 22, 2011

\begin{abstract}
Modern portfolio theory focuses on the relationship between risk and return, assuming away ambiguity, uncertainty over the probability space. This paper assumes that ambiguity affects asset prices and tests the relationship between risk, ambiguity and return based on a model developed by Izhakian (2011). Its contribution is twofold; it proposes an ambiguity measure that is derived theoretically and computed from stock market prices. Second, it uses ambiguity in conjunction with risk to test the basic relationship between risk, ambiguity and return. This paper finds that ambiguity has a consistently negative effect on returns and risk mostly has a positive effect.

1 mbrenner@stern.nyu.edu, Department of Finance, Stern School of Business, New York University, New York, NY, 10012, USA, +1-212-998-0323.

2 yud@stern.nyu.edu, Department of Economics, Stern School of Business, New York University, New York, NY, 10012, USA, +1-212-998-0017.

† The previous version of this paper was titled "Asset Prices and Ambiguity". We benefited from discussions with Yakov Amihud, John Asker, Doron Avramov, David Backus, Azi Ben-Rephael, Simon Benninga, Jacob Boudoukh, Adam Brandenburger, Ilan Cooper, Rob Engle, Itzhak Gilboa, Eitan Goldman, William Greene, Sergiu Hart, Clifford Hurvich, Saggi Katz, Ilan Kremer, Jacob Oded, Efe Ok, David Schmeidler, Yossi Spiegel, Marti Subrahmanyam, Avi Wohl, Stanley Zin and the seminar participants at New York University, the Interdisciplinary Center (IDC), Herzliya and The Hebrew University of Jerusalem.
\end{abstract}




\title{
Asset Pricing and Ambiguity: Empirical Evidence
}

\begin{abstract}
Modern portfolio theory focuses on the relationship between risk and return, assuming away ambiguity, uncertainty over the probability space. This paper assumes that ambiguity affects asset prices and tests the relationship between risk, ambiguity and return based on a model developed by Izhakian (2011). Its contribution is twofold; it proposes an ambiguity measure that is derived theoretically and computed from stock market prices. Second, it uses ambiguity in conjunction with risk to test the basic relationship between risk, ambiguity and return. This paper finds that ambiguity has a consistently negative effect on returns and risk mostly has a positive effect.
\end{abstract}

JEL Classification Codes: D51, D81, G12.

Key Words: Ambiguity, Ambiguity measure, Knightian uncertainty, Equity premium. 


\section{Introduction}

The fundamental relationship between risk and return of the market portfolio in the mean-variance paradigm is given by the following equation

$$
\mathrm{E}\left[r_{m}\right]-r_{f}=\gamma\left(\sigma_{m}^{2}\right)
$$

where $r_{m}$ is the return on the market portfolio, $r_{f}$ is the risk free rate, $\sigma_{m}^{2}$ is the risk of the market portfolio and $\gamma(\cdot)$ is a measure of risk aversion of a representative agent (or, an aggregation of risk aversion coefficients of investors). This linear relationship has been subjected to several time series empirical tests. Merton (1980) and French, Schwert and Stambaugh (1987) are two classic examples of studies that conducted such tests. While Merton (1980) focuses on estimation issues with the expected market return, French at al. (1987) focus more on alternative measures of risk (volatility). In general, the tests of the risk-return relationship have low $R^{2}$ and some of these tests result in negative coefficients of absolute risk aversion.

We believe that a missing factor that determines the expected excess return presented in equation (1) is ambiguity (the so called Knightian uncertainty) and the attitude towards it. Though there is an abundance of research on various aspects of ambiguity and ambiguity aversion, there is almost no empirical work providing a measure of ambiguity and incorporating such a measure in tests of the relationship between risk and return.

In this paper we introduce a measure of ambiguity, which is an additional factor determining the expected excess market return (also termed the equity premium). Equation (2) below is the expanded version of Equation (1) incorporating ambiguity. That is,

$$
\mathrm{E}\left[r_{m}\right]-r_{f}=\gamma\left(\sigma_{m}^{2}\right)+\eta\left(\mho^{2}\right),
$$

where $\mho^{2}$ measures the degree of ambiguity and $\eta(\cdot)$ is a measure of investors' attitude toward ambiguity. This measure is an outcome of the theoretical model developed by Izhakian (2011). The results are highly significant, challenging the conventional wisdom on investors' attitudes towards ambiguity. To the best of our knowledge, our study is the first empirical study that uses market data to measure ambiguity based on a theoretically derived model that combines risk and ambiguity. 
Modern portfolio theory, until recently, has practically ignored the Knightian distinction between risk and uncertainty. There were some exceptions like the collection of papers in a book edited by Bawa, Brown and Klien (1979). These papers, however, focus on estimation risk, how to correct for it, and how to incorporate it in portfolio selection or how it may affect capital market equilibrium. They did not deal with ambiguity and how it may affect asset prices and the relationship between ambiguity and return. Should ambiguity be priced? Can we separate risk and risk attitudes from ambiguity and attitudes toward ambiguity? How can we measure ambiguity? These are questions that, to the best of our knowledge, are still open and, in this paper, we try to deal with them.

In recent years there is a surge in research that tries to incorporate Knightian uncertainty naming it ambiguity or 'model risk'. For example, Uppal and Wang (2003), Epstein and Schneider (2008) and Ju and Miao (2011) support the model by calibration to the data. Anderson, Ghysels and Juergens (2009) and Dreschsler (2010), use proxies for 'model risk' like disagreement among analysts. Our approach is different, we measure ambiguity using market data.

The paper by Izhakian (2011) provides the theoretical underpinning of our paper which focuses on issues of ambiguity measurement and tests of risk-ambiguity-return relationships. In his paper Izhakian (2011) introduced a novel model of ambiguity, called Shadow probability theory (henceforth Shadow Theory) and studied how it affects investors' choices. The model provides a measure for the degree of ambiguity which is the center piece of the empirical tests that we employ in this paper. We focus on testing the effect of ambiguity on asset prices in a time series context while using the S\&P500 index as a proxy for the market portfolio. Our empirical results show that this measure has a significant effect on stock market returns.

We assume a representative investor whose reference point is zero excess-return. Assets' excess-returns are classified as gains or losses. Excess returns lower than zero are considered a loss and excess-returns equal or higher than zero are considered a gain. All assets' excess returns are assumed to be normally distributed. However, the parameters governing the distributions, i.e., mean and variance, are unknown and assumed to be random.

We show that, ambiguity and the excess-return on the market portfolio (the equity premium) are negatively correlated which implies that the degree of ambiguity is taken into 
account by investors when they price financial assets. It also implies that the representative investor, who holds the market portfolio exhibits ambiguity loving.

What is the evidence regarding investor's attitude toward ambiguity? It turns out that it depends on the states of nature that the investor faces. There is some evidence that an investor who faces a high probability of losses tends to embrace ambiguity, while if he faces a high probability of gains he may be ambiguity averse. Viscusi and Chesson (1999) found that people exhibit 'fear' effects of ambiguity for small probabilities of suffering a loss and 'hope' effects for large probabilities of loss. ${ }^{1}$ Considering investors in the stock market, where the probability of loss is relatively high (around 50\%), one would expect to observe ambiguity loving. Ivanov (2011) shows that more individuals exhibit ambiguity loving than ambiguity aversion. In particular, 32\% are classified as ambiguity-loving, compared to $22 \%$ who are classified as ambiguity averse, the remaining 46\% are considered ambiguity neutral. Assuming risk neutrality, Maffioletti and Michele (2005) also found ambiguity seeking in individuals' trading behavior. Analyzing statistical information of probabilities about health insurance, Wakker, Timmerman and Machielse (2007) document that individuals are ambiguity seeking. In an experimental study of bidders' behavior Chen, Katušcák and Ozdenoren (2007) suggests that individuals are ambiguity seeking. In general, most behavioral studies find ambiguity loving behavior when there is a relatively high probability of suffering a loss.

Consistent with the above studies, our results show that investors are ambiguity lovers. In our study the average probability of loss is relatively high (almost 50\%) and as found by Viscusi and Chesson (1999), in such cases, investors are ambiguity loving. These findings are consistent with our theoretical model. When returns are symmetrically distributed, an investor who maximizes expected return minimizes the probability of loss. Therefore, when the probability of loss is relatively high, given two assets with identical risk, he prefers the asset with the random probability over an asset with known probabilities. Such preferences imply ambiguity loving.

The rest of the paper is organized as follow. Section 2 provides the theoretical framework. Section 3 discusses the data and the empirical methodology. Section 4 presents the empirical findings. Section 5 discusses the findings regarding investors' attitudes toward risk and ambiguity and Section 6 provides summary and conclusions.

\footnotetext{
${ }^{1}$ Abdellaoui, Baillon, Placido and Wakker (2011) tie ambiguity loving to the source of ambiguity.
} 


\section{The theoretical model}

Recently, Izhakian (2011) introduced a novel model of ambiguity, called Shadow theory, which provides a measure of the degree of ambiguity. This measure is the center piece of the empirical tests that are conducted in this paper. Next we provide a detailed summary of the main principles of Shadow theory and how we use it in the empirical tests.

\subsection{Preliminaries}

The theory of financial assets prices is mainly based on the expected utility (von Neumann-Morgenstern (1944) and Savage (1961)) paradigm, which assumes that decision makers know, or act as if they know, the probabilities of all states of nature. A basic issue with these models is that in reality the investor does not know the precise probabilities of events (see Ellsberg (1961)), which means that individuals are exposed not only to risk but also to ambiguity (Knightian uncertainty). Several models that deal with decision making under uncertainty have been suggested. These include the subjective nonadditive probabilities of Gilboa (1987), the Choquet expected utility (CEU) of Schmeidler (1989), the multiple prior (MEU) of Gilboa and Schmeidler (1989), the model misspecification of Hansen and Sargent (2001) and non-reducible second-order probabilities models of Segal (1987) and Klibanoff, Marinacci, and Mukerji (2005)). ${ }^{2}$ While this literature made a considerable contribution to understanding the decision maker's preferences toward ambiguity, a complete separation between ambiguity and risk, which enables to measure ambiguity empirically, has not been derived. Such a measure is necessary in testing the effect of ambiguity on asset prices.

Shadow Theory provides a measure of ambiguity. It assumes that probabilities of observable events, are random and are dominated by unobserved, with a second-order probability. In this framework a complete separation between risk and ambiguity and between preferences and beliefs is obtained. This allows us to measure the degree of ambiguity. In this model, random probabilities are subjectively interpreted by decision makers in a nonlinear way, characterized by probabilistic sensitivity to ambiguity. Ambiguity aversion, thus, takes the form of subadditive (subjective) probabilities (i.e., the probabilities add up to number smaller than 1).

\footnotetext{
${ }^{2}$ Other models that relax the reduction between first and second order probabilities include Klibanoff et al. (2009), Ju and Miao (2011), Hayashi and Miao (2011), Ergin and Gul (2009), Nau (2006), and Chew and Sagi (2008).
} 
In this context, when ambiguity is present, ambiguity loving implies a superadditive probability measure (i.e., the probabilities add up to number greater than 1).

The Shadow theory developed in Izhakian (2011) extends the Choquet expected utility of Schmeidler (1989) and Tversky and Kahneman's (1992) cumulative prospect theory. ${ }^{3}$ Using Wakker's axioms (Wakker 2010) it models reference-dependent beliefs in a two-sided Choquet expected utility framework for losses and for gains, separately. Shadow theory assumes that the financial decision maker (henceforth DM or investor) has a reference point that separates losses from gains. Outcomes that are lower than this reference point are considered a loss and outcomes which are higher than the reference points are considered a gain. The reference point in Izhakian (2011) serves as the reference that separates the probabilities of gains from the probabilities of losses. The volatility of these probabilities is used in measuring the degree of ambiguity. Attitudes toward ambiguity are formed with respect to this degree of ambiguity.

The implication of a subadditive probability measure for asset prices is that there is an ambiguity premium in addition to the conventional risk premium. The conventional risk premium is the premium that a DM is willing to pay for replacing a risky bet by its expected outcome. The ambiguity premium is the premium that a DM is willing to pay for replacing an ambiguous bet by a risky, non ambiguous, bet with an identical expected outcome. The uncertainty premium is the total premium that a DM is willing to pay for replacing an ambiguous bet by its expected outcome, i.e., it contains both, a risk premium and an ambiguity premium.

Let $r$ be the random return on an asset, $\mathrm{U}(\cdot)$ be the utility function, for risk and $\psi(\cdot)$ be the sake function for ambiguity. The uncertainty premium is provided by

$$
K=\underbrace{-\frac{1}{2} \frac{\mathrm{U}^{\prime \prime}(\mathrm{E}[r])}{\mathrm{U}^{\prime}(\mathrm{E}[r])} \operatorname{Var}[r]}_{R} \underbrace{-\frac{1}{4}\left[\frac{1}{2} \frac{\psi^{\prime \prime}\left(\mathrm{E}\left[\mathrm{P}_{L}\right]\right)}{\psi^{\prime}\left(\mathrm{E}\left[\mathrm{P}_{L}\right]\right)}+\frac{1}{2} \frac{\psi^{\prime \prime}\left(\mathrm{E}\left[\mathrm{P}_{G}\right]\right)}{\psi^{\prime}\left(\mathrm{E}\left[\mathrm{P}_{G}\right]\right)}\right] \mho^{2}[r]}_{A},
$$

where $R$ is the risk premium and the second component, $A$, is the ambiguity premium. The parameters $\mathrm{P}_{L}$ and $\mathrm{P}_{G}$ are the random probabilities of loss and of gain, respectively. The

\footnotetext{
${ }^{3}$ Cumulative prospect theory, developed by Tversky and Kahneman (1992), generalizes the original prospect theory by Kahneman and Tversky (1979). It modifies the probability weighting functionals of the original prospect theory, such that it always satisfies stochastic dominance and supports an infinite state space.
} 
expectation $\mathrm{E}\left[\mathrm{P}_{L}\right]$ and $\mathrm{E}\left[\mathrm{P}_{G}\right]$ are taken with respect to the likelihoods of the possible probability measures; i.e. with respect to the second-order probabilities. That is,

$$
\mathrm{E}\left[\mathrm{P}_{L}\right]=\sum_{i=1}^{M} \chi_{i} \mathrm{P}_{i}\left(r<r_{k}\right) \quad \text { and } \quad \mathrm{E}\left[\mathrm{P}_{G}\right]=\sum_{i=1}^{M} \chi_{i} \mathrm{P}_{i}\left(r \geq r_{k}\right)
$$

where $r_{k}$ the reference return which distinguishes losses from gains and $\chi_{i}$ is the probability of the probability distribution $\mathrm{P}_{i}$.

The expected return is $\mathrm{E}[r]$, where the expectation of the outcome is evaluated using the expected probabilities for each outcome. It combines two expectations; with respect to the random outcomes and with respect to the random probabilities. The parameter

$$
\mho^{2}[r]=4 \operatorname{Var}\left[\mathrm{P}_{L}\right]
$$

is Izhakian's measure of ambiguity, which is four times the variance of the probability of loss or four times the variance of the probability of gain, which are taken with respect to the second order probability distribution $\chi \cdot{ }^{4}$ It is important to note that, $\mho^{2} \in[0,1]$, attains its minimum value, 0 , when all probabilities are known, and its maximum value, 1 , only in the extreme case of binomial distribution with a random probability for each event that can have probabilities of 0 or 1 with equal chances. The Arrow-Pratt coefficient of absolute risk aversion is $-\frac{\mathrm{U}^{\prime \prime}(\cdot)}{\mathrm{U}^{\prime}(\cdot)}$. The coefficient of absolute ambiguity aversion is $-\frac{\psi^{\prime \prime}(\cdot)}{\psi^{\prime}(\cdot)}$.

Equation (3) defines the premiums required by investors for bearing risk and ambiguity associated with holding the asset.

\subsection{Intuition}

To provide some intuition with regard to the measure of ambiguity, $\mho^{2}[r]$, lets consider the following binomial example. Assume an asset with the following two possible future returns $d=-10 \%$ and $u=20 \%$. Consider the case where the probabilities of $d$ and $u$ are known, say

\footnotetext{
${ }^{4}$ This equality is obtained since the variance the probability of an event is equal to the variance of the probability of its complement event.
} 
$\mathrm{P}(d)=\mathrm{P}(u)=0.5$. The expected return is, thus, 5\%. Taking the standard deviation of outcomes, in terms of return, as a proxy for the degree of risk, is $15 \%$. Obviously, since the probabilities are precisely known, ambiguity is not present and the investors face only risk.

Assume now that the probabilities of $d$ and $u$ can be either $\mathrm{P}(d)=0.4$ and $\mathrm{P}(u)=0.6$ or alternatively $\mathrm{P}(d)=0.6$ and $\mathrm{P}(u)=0.4$, where the two possible distributions are equally likely. This means that the investors are now facing not only risk but also ambiguity. The main idea of the measure of ambiguity, $\mho^{2}[\cdot]$, is that, similar to measuring the degree of risk by the variance of outcomes, we can measure the degree of ambiguity by the variance of probabilities. However, concerning the variance of probabilities, the question is; to the probability of which event is the variance applied. The natural choice would be the probability of the cumulative event of gain or the probability of the cumulative event of loss, for which the variance is identical since the event of loss is the complement of the event of gain and the objective probabilities are additive. Computing the variance of the probability of loss yields $\operatorname{Var}\left[\mathrm{P}_{L}\right]=0.01$ which in turn indicates a degree of ambiguity of $\mho=0.2$. Notice that the degree of risk has not changed since the variance is computed using the expected probabilities $\mathrm{P}(d)=\mathrm{P}(u)=0.5$.

\subsection{The risk-ambiguity model}

Assume an economy in which the returns on all assets are normally distributed. The return on the market portfolio, $r_{m}$, is, therefore also normally distributed. The representative investor in this economy uses the risk free rate, $r_{f}$, as the reference point relative to which he classifies outcomes as a loss or a gain. That is, any return on the market portfolio lower than $r_{f}$ is considered a loss and any return higher than $r_{f}$ is considered a gain. Formally, the probability of loss takes the form

$$
\mathrm{P}_{L}=\operatorname{Pr}\left[r_{m} \leq r_{f}\right]=\int_{-\infty}^{r_{f}} \frac{1}{\sqrt{2 \pi \sigma^{2}}} e^{-\frac{\left(r_{m}-\mu\right)^{2}}{2 \sigma^{2}}} d r_{m}=\Phi\left(\frac{r_{f}-\mu}{\sigma}\right)
$$


where $\Phi(\cdot)$ stands for the standard normal cumulative probability distribution. Recall, that in Shadow theory when ambiguity is present the variable $\mathrm{P}_{L}$ is random since the normal probability distribution is governed by the random parameters $\mu$ and $\sigma$. We assume that ratio of these two parameters, $\mu$ and $\sigma$, is normally distributed and that $\mathrm{E}\left[\mathrm{P}_{L}\right] \approx \mathrm{E}\left[\mathrm{P}_{G}\right]$. To allow tractability, and without loss of generality, the representative investor in our economy exhibits constant absolute risk attitude (CARA) and constant absolute ambiguity attitude (CAAA) ${ }^{5}$. The uncertainty premium, defined by Equation (3), is thus simplified to

$$
K=\gamma \frac{1}{2} \operatorname{Var}\left[r_{m}\right]+\eta \frac{1}{4} \mho^{2}\left[r_{m}\right]
$$

where

$$
\mho^{2}\left[r_{m}\right]=4 \operatorname{Var}\left[\Phi\left(\frac{r_{f}-\mu}{\sigma}\right)\right]
$$

$\gamma$ is the coefficient of the investor's risk aversion and $\eta$ is the coefficient of ambiguity aversion. A positive (negative) $\gamma$ implies risk aversion (risk seeking), while a positive (negative) $\eta$ implies ambiguity aversion (ambiguity seeking).

The expected return on the market portfolio, $r_{m}$, less the risk free rate, also called excess return, thus takes the form

\footnotetext{
${ }^{5}$ Though we assume CRRA for risk, we assume CAAA for ambiguity. The literature usually documents CRRA for investors, see for example Kachelmeier, and Shehata.(1992), Chetty (2006), Schechter (2007) and Cohen and Einav (2007). CRAA means that the impact of the attitude toward ambiguity on the subjective probabilities of an event is decreasing with its expected probability. That is, the subjective probabilities of highly likely events are less affected by individuals' attitude toward ambiguity. Whereas, CAAA means that the impact of the attitude toward ambiguity on the subjective probabilities of an event is independent of its expected probability. We find that CAAA is more reasonable. Technically, the subjective probability of event $j$ takes the form $Q_{j}=\mathrm{E}\left[P_{j}\right]+\frac{1}{2} \frac{\psi^{\prime \prime}\left(\mathrm{E}\left[P_{j}\right]\right)}{\psi^{\prime}\left(\mathrm{E}\left[P_{j}\right]\right)} \zeta_{j}^{2}$, where $\mathrm{E}\left[P_{j}\right]$ is the expected probability of event $j, \frac{\psi^{\prime \prime}\left(\mathrm{E}\left[P_{j}\right]\right)}{\psi^{\prime}\left(\mathrm{E}\left[P_{j}\right]\right)}$ is the coefficient of ambiguity attitude and $\zeta_{j}^{2}$ is the degree of ambiguity of event $j$ measured by the variance of the probability, see Izhakian (2011). Therefore, for CRAA, the subjective probability is $Q_{j}=\mathrm{E}\left[P_{j}\right]-\frac{\eta}{2 \mathrm{E}\left[P_{j}\right]} \zeta_{j}^{2}$ and for CAAA the subjective probability is $Q_{j}=\mathrm{E}\left[P_{j}\right]-\frac{1}{2} \eta \zeta_{j}^{2}$
} 


$$
\mathrm{E}\left[r_{m}\right]-r_{f}=\gamma \frac{1}{2} \operatorname{Var}\left[r_{m}\right]+\eta \frac{1}{4} \mho^{2}\left[r_{m}\right]
$$

where the risk premium is $R=\gamma \frac{1}{2} \operatorname{Var}\left[r_{m}\right]$ and the ambiguity premium is $A=\eta \frac{1}{4} \mho^{2}\left[r_{m}\right]$. The effect of uncertainty on the return is now represented by two terms; a risk term and an ambiguity term. Each is measured separately and has a different effect on the excess return. In the next section we present the empirical tests of this model. We first provide the methodology that we use to measure the variables, especially the ambiguity measure, and then we apply the model to empirical tests.

\section{Data and methodology}

\subsection{Data}

The main body of data used in the empirical research is intraday trading data (prices and volumes) on the exchange-traded fund SPDR (Ticker: SPY) taken from the TAQ database. ${ }^{6}$ The Standard \& Poor's Depositary Receipts (SPDR) is comprised of all the stocks in the Standard \& Poor's 500 Index. The stocks in the SPDR have the same weights as in the index and it is designed to track the index, before expenses. The expense ratio is about 7-8 basis points and the bid-ask spread is 1-2 basis points. The quarterly dividends are added to the index every 3 months. It can be sold short like any other stock and short interest is sometimes as high as 50 percent. A typical volume for the SPDR is between 200- 300 million shares per day, which is the highest of any US stocks traded on any exchange.

We use the SPDR as a proxy for the market portfolio and not the S\&P index itself since the SPDR trades continuously, while the index contains illiquid stocks and so its values are stale. The data covers the period from February 1993 to December 2010. ${ }^{7}$ Monthly returns adjusted for

\footnotetext{
${ }^{6}$ The Trade And Quotes (TAQ) database; Wharton Research Data Services (WRDS).

${ }^{7}$ Under the ticker symbol, SPY, SPDRs began trading on the American Stock Exchange (AMEX) on January 29, 1993.
} 
dividends obtained from the CRSP database. ${ }^{8}$ VIX values were obtained from the CBOE site, and the risk free rate from Ibbotson Associates. ${ }^{9}$

\subsection{Methodology}

The first step in designing the empirical tests is to compute the time series values of the variables that will be used in the tests. We first compute the degree of ambiguity derived by Izhakian (2011, Equation (8)) for each period of one month. ${ }^{10}$ We sample the prices of SPY every 15 minutes starting from 9:30 until 16:00 each day: 27 prices in total for each day. ${ }^{11,12}$ In case there was no trade at a specific sampling time, we took the volume weighted average value of the closest trading prices. Using these prices we compute 15 minute returns, 26 returns for each day. ${ }^{13}, 14$ The choice of 15 minute intervals is dictated by the measure of ambiguity. To perform meaningful time series tests, in our 18 year period (1993 to 2010), we need to use monthly observations. To obtain a statistically meaningful monthly measure of ambiguity we need a daily estimate of probability derived from a daily distribution of rates of return, which, in turn, requires intraday observations. Anderson, Bollerslev, Diebold and Ebens (2001) show that computing returns using 5-minutes time-intervals eliminates microstructure effects. Furthermore, since the SPY is frequently traded its bid-ask spread is minimal, such that these returns are not biased.

For each day we used its 26 observations to compute the mean and the variance of return. Depending on the number of trading days in the month, we have, for each month, between 440

\footnotetext{
${ }^{8}$ Since dividends are added to SPDR every three months, we adjust the return on SPDR, the explanatory variable, to monthly dividend yields, using the dividend yields on the S\&P-500 index, taken from the CRSP database.

${ }^{9}$ The risk free rate is one-month Treasury bill rate of return (from Ibbotson Associates).

${ }^{10}$ For simplicity we concentrate on one month intervals, however the same procedure can be applied for periods of less than one month, 10 trading days for example.

${ }^{11}$ We also test our model using a 10 minutes interval; the results were essentially the same.

${ }^{12}$ To check for robustness, while eliminating the impact of the trading noise caused by opening and closing daily positions during first and the last half-hour of the stock trading, we also performed our tests using only the prices from 10:00 to 15:30. The results were essentially the same.

${ }^{13}$ We have not included returns between closing prices and opening prices of the following day. We eliminated the impact of overnight price changes and dividend distributions.

${ }^{14}$ While omitting the first and last half an hour of the trading our results remain almost similar.
} 
and 572 observations. ${ }^{15}$ Using Equation (6) we compute for each day the probability to suffer a loss, $\mathrm{P}_{L}$. For each month, there is a vector which consists of 20 to 22 different loss-probabilities. Using this vector of loss-probabilities we compute its variance to obtain the degree of ambiguity, $\mho^{2}$, for that month. Assuming that the daily ratio $\lambda=\frac{\mu}{\sigma}$ is normally distributed with mean $E[\lambda]$ and variance $\operatorname{Var}[\lambda]$ then $P_{L}$ is uniformly distributed across the month. ${ }^{16}$ This method assigns lower weights to values of $\lambda$ that deviate from the monthly mean $\lambda$. To estimate the expected probability of loss we use the realized probabilities of loss as a proxy for the expected. These probabilities are computed using daily means and variances, $\mu$ and $\sigma$ computed from 15 minute intervals and their ratio, $\lambda=\frac{\mu}{\sigma}$, ranges between 0.72 and -0.83 . Since this ratio is distributed normally, extreme values of daily $\lambda$ will get very little weight in the monthly estimate of $\mu$ and $\sigma$ of $\lambda$. The variation of the probability of loss, $\mathrm{P}_{L}$, is due to the variation of the ratio $\lambda$. A closer look at the variation of this ratio shows that it is mainly driven by the variation of $\sigma$. Over the entire sample the standard deviation of $\sigma$, in terms of daily return, is $0.641 \%$ while the standard deviation of $\mu$ is only $0.211 \%{ }^{17}$

The risk factors are estimated from the daily variances over the month. We compute the mean of the variance, MVAR, and the variance of variance VVAR for every month. As controls, for each day we also compute the skewness and the kurtosis, and for each month the average skewness (MSKW) and the average kurtosis (MKRT). Using these variables, we next test the risk

\footnotetext{
${ }^{15}$ To check for robustness, we formed randomly (without repetition) groups of 26 observations and computed a mean and a variance for each group. Since the results of this method were not significantly different from the first method, we conducted our tests using the first method.

${ }^{16}$ It can be shown that the density function of the random variable $\mathrm{P}_{L}$ as a function of the normally distributed random variable $\lambda$ is uniform.

${ }^{17}$ We would like to emphasize that mean realized returns measured over short intervals are very poor proxies for annual expected return (i.e. their standard error is very large). In our context, however, we derive the daily probability of loss from a distribution of the ratio of $\mu$ and $\sigma$, which gives very little weight to extreme observations. This results in very reasonable estimates of the expected probability of loss. On the average the probability of loss should be lower than 50\%. In our sample the average probability of loss is $49.75 \%$, even though in some days the realized probability of loss exceeded the $50 \%$. The standard deviation of the probability of loss over the entire sample was $1.5 \%$.
} 
and ambiguity effect on monthly returns, i.e., Equation (9) is subjected to regression tests presented in the next section.

\subsection{Descriptive statistics}

The dependent variable is the monthly return on SPY, which serves as a proxy for the return on the market portfolio, $r_{m}$, minus the risk free rate, $r_{f}$, which is the 1 month T-bill rate. The market return, $r_{m}$, is computed using the opening price on the first trading day of the month and the closing price on the last trading day of that month, and it is adjusted for monthly dividends. The other variables that we use in the empirical tests are as follows: MVAR is the mean of daily variance. The daily variance is computed every day using 15 minutes rates of return (ROR) and multiplied by 26 (the number of 15 minutes intervals). It is averaged over the number of trading days in a month. VVAR is the variance of daily variance, computed by using the daily variances during the month. MSKW is the mean of daily skewness, computed every day using 15 minute ROR, multiplied by $26^{3 / 2}$ and averaged over the month. MKRT is the mean of daily kurtosis, calculated every day using 15 minute ROR multiplied by $26^{2}$ and averaged over the month. $M V I X^{2}$ is the mean of all daily squared VIX observation during the month. VVIX ${ }^{2}$ is the variance of all daily squared VIX observation during the month. CVAR is the variance of 15 minutes return of the last day in the month (converted to daily). CVIX ${ }^{2}$ is the closing squared VIX on the last day of the month (converted to daily). DVAR is the change in variance from the last day in month $t-1$ to last day in month $t$. DVIX ${ }^{2}$ is the change in squared VIX from the last day in month $t-1$ to last day in month $t$.

Table I panel A provides summary statistics of the variables that are used in the empirical tests. All variables are adjusted to daily terms. During the 1993-2010 period, the daily mean return on SPY, $r_{m}$, is $0.025 \%$, about 9.2 percent on an annual basis. The variance of $r_{m}$ is about 3.0E-06. The risk free rate, $r_{f}$, is $0.009 \%$, about 3.33 percent annually. The excess return, $r_{m}-r_{f}$, is $0.016 \%$, 5.84 percent annually. The distribution of $r_{m}-r_{f}$ is somewhat negatively skewed. Most values, however, are to the right of the mean. The positive kurtosis, 0.995, is an indication of fat tails.

The average daily variance (across all 215 months), MVAR, is about a half of the average daily $\operatorname{VIX}^{2}(0.00012$ vs. 0.0002$)$, while the variance of these two estimates is about the same 
(4.14E-08 vs. 4.00E-08). (the average standard deviation on an annual basis is about 14 percent while VIX is about 21). In the same vain, the average variance of the daily variance, VVAR, is about 8.11E-06 percent while the average variance of the daily VIX is only about 4.65E-09. Thus, VIX is on the average larger than the realized volatility by about 6-7 percent, but it moves in a narrower range. ${ }^{18} \mathrm{MSKW}$ is the mean of the daily skewness, which is negative but not significant. MKRT is the mean of the daily kurtosis, which is significantly different from zero, indicating fat tails as observed earlier.

Panel B in Table I provides summary statistics of the ambiguity parameters. The average daily probability of loss, $\mathrm{E}\left[P_{L}\right]$, is 49.7 percent. $\mathrm{E}\left[P_{L}\right]$ is computed using Equation(4). The distribution of these probabilities is positively skewed, 0.054, and has thin tails (the kurtosis is 0.596). The measure of ambiguity, $\mho^{2}$, given in Equation (8), is on the average 0.025, where the minimum and maximum observed values are 0.0088 and 0.0532 respectively. $\mho$ (the square root of the measure $\mho^{2}$ ) the daily ambiguity level measured in percentage points, is 15.6. Figure 2 depicts the distribution of $\mho$ in the period 1993 to 2010. The distribution of $\mho$ is almost symmetric around its mean of $16 \%$.

Since our model predicts that the excess return should be affected by the ambiguity measure $\mho^{2}$ in a linear manner, we use $\mho^{2}$, rather than $\mho$, in our tests. Table I, panel B, shows that $\mho^{2}$ is positively skewed, with coefficient 0.6832 , which is highly significant. Recall that the ambiguity measure takes on only positive values in the range between 0 and 1 . The positive skewness thus indicates that the ambiguity level is usually concentrated around the mean with a long tail, where in some months we observe a relatively high level of ambiguity. The kurtosis of $\mho^{2}$ is slightly positive, $0.2437 .{ }^{19}$

To get a more intuitive feel for the measure of ambiguity we can look at $\mho$ (not $\mho^{2}$ ). During the period 1993 to 2010, the mean level of ambiguity, $\mho$, is about 15.6 percent, while its

\footnotetext{
${ }^{18}$ The variance of MVAR is the variance of the monthly average variance calculated for each day separately. The variance of the parameter VVAR is the variance of the monthly variance base on the intraday variance.

${ }^{19}$ Since we also test the case of constant relative ambiguity aversion (CRAA), the summary statistics of the normalized, relative ambiguity measure, $\mho / \mathrm{E}\left[P_{L}\right]$ are also presented in Table I. One can see the relative ambiguity is positively skewed, 12.5951 , but with negative kurtosis, -0.4084 , which indicates thin tails.
} 
standard deviation is about 2.5\%. The lowest recorded level of ambiguity is $9.3 \%$ and the highest is $23.1 \%$ (December 2002, when the US decided to invade Iraq).

In Panel C, of table I, we provide summary statistics of CVAR, CVIX ${ }^{2}, D V A R, D V I X^{2}$. $C V I X^{2}$, the value of $\mathrm{VIX}^{2}$ on the last day of the month is practically the same as $M V I X^{2}$ but the variance is lower since $C V I X^{2}$ does not include some extremely large observations. The same is true for CVAR and MVAR.

\section{[ INSERT TABLE I ]}

Table II provides the first 6 autocorrelation coefficients, of all the variables that we use in our tests. The variables that have large and significant autocorrelations are those who use VIX in their various forms, except for $D V I X^{2}$. For example, $M V I X^{2}$ has a $1^{\text {st }}$ order autocorrelation of 0.85 and it decays slowly to 0.29 at the $6^{\text {th }}$ order. A similar pattern is observed for VVIX ${ }^{2}$ and $C V I X^{2}$. The main concern is how it may affect the OLS estimator in our regression tests. We therefore conducted first the tests proposed by Amihud and Hurvich (2004) and then used the regression test.

\section{[ INSERT TABLE II ]}

Figure 1, the upper plot describes the average daily excess returns on the SPDR (SPY) over the years 1993 to 2010. In the lower plot we present the monthly ambiguity, $\mho$, on a daily basis. Over this period we observe only a couple of months that contain big downward moves in the market. The two obvious ones, are September of 1998, the Russian default and the LTCM debacle, and September 2008, the recent financial crisis. It seems that during the 1990s ambiguity levels were not very high, but they have increased by at least $50 \%$ after 2000 . It can be observed that relatively low returns are accompanied by relatively high levels of ambiguity in the previous month. For example, on August 1998 the excess return dropped to $-0.48 \%$ and a month before, July 1998, the ambiguity level, $\mho$, jumped to 0.182. Or in September 2002, the return on SPY dropped to $-0.36 \%$ while the level of ambiguity in the month before jumped up to 0.184 . On

September 2008 the return on SPY dropped to the low $-0.54 \%$, where in the months before ambiguity jumped to a level higher than 0.197 . It is interesting to note that over the entire period ambiguity and excess return seem to move counter cyclically (depicted by the solid lines).

\section{[ INSERT FIGURE 1 ]}


Figure 2 describes the distribution of the degree of ambiguity, $\mho$, in the period between February 1993 to December 2010. The degree of ambiguity is provided on the x-axes in percentages. The y-axes describes the frequency of the degree of ambiguity. Most of the observations are centered between $12 \%$ and $20 \%$ ambiguity. There are a few cases where the degree of ambiguity is higher than $20 \%$ or lower than $10 \%$, which is very rare.

\section{[ INSERT FIGURE 2 ]}

Table III provides the cross correlations of all variables used in this study. It provides a first look at the relationships between all possible pairs of variables. In particular, the relationship of the excess return, $r_{m}-r_{f}$, and the ambiguity measure $\mho^{2}$. It is negatively correlated and significant, which indicates that the coefficient of ambiguity is possibly negative. Or, in other words, that investors love ambiguity. Also, the ambiguity measure exhibits a low correlation with the other variables. This basically rules out the possibility that ambiguity is a proxy for volatility of volatility or kurthosis. The various measures of volatility exhibit some correlation but those are not large enough to affect our main tests.

\section{[ INSERT TABLE III ]}

\section{Empirical results: testing the effect of ambiguity and risk on excess returns}

Theoretically, the effect of ambiguity and risk on the expected excess returns, presented in Equation (9), assumes that investor exhibit constant relative risk aversion (CRRA) and constant absolute ambiguity aversion (CAAA). ${ }^{20}$ In table IV we present the results of the regression tests where the dependent variable is the excess return and ambiguity and risk are the independent variable. We assume that the observed excess return is the best estimate of the expected excess return and so it is for the other variables like measures of expected risk by the daily variance.

In table IV the independent variables are measured contemporaneously with the excess return. ${ }^{21}$ So the return in month $t$ is explained by the ambiguity in month $t$, by the variance in

\footnotetext{
${ }^{20}$ We also tested our model for the case of constant relative ambiguity attitude (CRAA). The results were not significantly different than the results for the CAAA case.

${ }^{21}$ The values of the Durbin-Watson (DW) test indicate that we don't have a serial correlation issue.
} 
month $t$, etc. We first used only ambiguity to explain the excess return and found that the ambiguity effect is negative and highly significant. When MVAR and VVAR were included, the $R^{2}$ has increased from 5\% to $18 \%$ but we were puzzled by the sign and significance of the MVAR coefficient, which was negative while we expected MVAR to be positive. Since these realized values may be poor proxies for expected variance and variance of variance, $M V I X^{2}$ and $V V I X^{2}$ were used as estimates of the expected risk measures. $M V I X^{2}$ turns out to be non significant but $V V I X^{2}$ is negative and significant. We also included a measure of Kurtosis, denoted MKRT, to see whether the measure of ambiguity is possibly a proxy for Kurtosis, which turns out to be non significant and does not affect the significance of ambiguity. ${ }^{22}$

The results in table IV show that ambiguity is an important variable in explaining excess returns and is not a proxy for other possible factors. However, we did not find our measures of risk to have the effect dictated by our fundamental paradigm that implies a positive relationship between risk and return. In general, past empirical studies have not provided conclusive evidence, especially the time-series tests. French, Schwert and Stambough (1987) is possibly the best known time series study that provides results that could be interpreted as supporting the basic theory, though they also come up with some mixed results. Since they only had a long time series of monthly data, we thought that the use of daily data (constructed from intraday data) may provide us with more promising results. In fact, our contribution is twofold. First, we argue that there is a missing variable, namely ambiguity. Second, we use data that are more fine-tuned to test the basic relationship between risk and return. In our tests we also argue that the measure of risk is orthogonal to the measure of ambiguity, which we observe in the low correlation of these two measures.

\section{[ INSERT TABLE IV ]}

The results in table IV which use ex post measures of risk (and ambiguity), are consistent with the tests and results in other studies on the relationship between risk and return (e.g. French, Schwert and Satmbaough (1987)). In table V we use the measures of risk at $t$-1, coinciding with the market price at $t-1$. We argue that the level of risk and ambiguity at $t-1$ affect the price at $t-1$ instantaneously and consequently the return from $t-1$ to $t$. The $t-1$ measures of risk and ambiguity

\footnotetext{
${ }^{22}$ We conducted the same test for skweness. We included a measure of skewness, denoted MSKW, to see whether the significance of the measure of ambiguity is affected by skewness. It turns out that skewness is not significant and does not affect the significance of ambiguity.
} 
could be considered ex ante (expected) estimates which should affect the return in time $t$. Since the explanatory variables are measured at $t-1$, it is likely that we obtain biased coefficients and Tstatistics due to a serial correlation. To deal with this issue, we subjected the regressions to the Amihud and Hurvitch (2004) test and all the regressions have "passed" the test, so no adjustments were necessary. ${ }^{23}$

The results in table $\mathrm{V}$ are encouraging. In all the regressions ambiguity is highly significant, while the risk measures have positive coefficients, though most of them are not significant. To measure risk in $t-1$ we have used $M V A R$, the mean daily variance and $M V I X^{2}$, the mean daily $\mathrm{VIX}^{2}$. To better align the measure of risk with the price at $t-1$, we have also used CVAR, the estimate of the variance on the last day of the month, adjacent to the opening price used for the return from $t-1$ to $t$. Similarly, the last regression on table V uses the closing VIX ${ }^{2}$, just before the opening price the next day. The best result is obtained when we include VVIX ${ }^{2}$, the volatility of $\mathrm{VIX}^{2}$, in the regression. Ambiguity is not affected; it is negative and as significant as in the other regressions. Expected volatility, estimated by $M V I X^{2}$, is positive with a coefficient of 2.6 and highly significant.

\section{[ INSERT TABLE V ]}

Our next set of tests, provided in table VI, further support the findings in table V. As suggested by French, Schwert and Stambough (1987), we have used the unexpected change in risk to explain the excess return, $r_{m}-r_{f}$. We measure this change in two ways; $D V A R_{t}$, the change in the daily variance, from $t-1$ to $t$, using the variance on the last trading day of the month. DVIX ${ }_{t}^{2}$, the change in daily VIX ${ }^{2}$, from $t-1$ to $t$, using $\mathrm{VIX}^{2}$ on the last trading day of the month. The results in this table are our strongest results. We use the ambiguity measure, $\mho^{2}$, in $t$ 1 to be consistent with the risk measures and can be considered an ex ante measure. As seen in the earlier tests, it is negative and highly significant. Both of the risk measures are also negative and highly significant as hypothesized. The regression which uses ambiguity and $D V I X^{2}$ provides

\footnotetext{
${ }^{23}$ The Amihud and Hurvitch (2004) test is applied when the explanatory variable is a lagged variable. The residual from the OLS regression (the main model) is regressed against the residual from the autoregressive regression of the explanatory variable. According to this test, the estimated statistics are biased only if both of the following two conditions are satisfied. (i) the explanatory variables are highly autocorrelated. (ii) there is a statistically significant correlation between the residuals of the autoregressive regression of the explanatory variable and the residuals of the main regression, explaining $r_{m}-r_{f}$ in our case. These two conditions have not been satisfied together in any of the regressions we tested.
} 
even stronger result, the $R^{2}$ is about 46 percent. The ambiguity measure turns out to be significantly negative in any specification of the determinants of excess return. As stated in the introduction, previous evidences regarding the attitudes of ambiguity are mixed at best. Our results are consistent with the studies that show ambiguity loving. The effect of risk, measured by the unexpected change in volatility, can be interpreted as "indirect evidence of a positive ex ante relation” (see French, Schwert and Stambough (1987, p. 4)).

\section{[ INSERT TABLE VI ]}

\section{Investors attitude toward risk an ambiguity}

Examining further the results in table $\mathrm{V}$ we see, for example, that in the regression with $M V I X_{t-1}^{2}$ and $V V I X_{t-1}^{2}$, the coefficient of ambiguity $\mho_{t-1}^{2}, \beta_{1}$ equals -0.0492 and the coefficient of risk ( $\left.M V I X^{2}\right), \beta_{4}$ equals 2.62. These results imply that the investor's coefficient of constant relative risk aversion is $5.24 .^{24}$ Though this number is in the range of estimates obtained in other studies (e.g. Brown and Gibbons (1985), French, Schwert and Stambough (1987)), it is on the high end indicating strong aversion to risk. The investors' coefficient of constant absolute ambiguity attitude, however, indicates that investors are typically ambiguity lovers characterized by a coefficient of ambiguity loving of -0.2. To the best of our knowledge, this is the first empirical study which provides an estimate of the degree of the attitude toward ambiguity.

At first it seems puzzling that investors exhibit risk aversion and ambiguity loving at the same time. To explain this puzzle let's assume two assets with identical expected return, but the first asset has a random probability of loss/gain and the second asset's probability of loss/gain is equal to the expected probability of loss/gain of the first asset. By definition, an ambiguity lover prefers the first asset over the second asset. In our setting, returns are normally distributed, yet with random mean and random variance, such that if

$$
\mathrm{E}[\mathrm{P}(L \mid \mu, \sigma)]=\mathrm{E}\left[\Phi\left(\frac{r_{f}-\mu}{\sigma}\right)\right] \leq \Phi\left(\frac{r_{f}-\mathrm{E}[\mu]}{\mathrm{E}[\sigma]}\right)=\mathrm{P}(L \mid \mathrm{E}[\mu], \mathrm{E}[\sigma]),
$$

\footnotetext{
${ }^{24}$ For completeness, we tested the impact of investors' loss aversion by controlling for different levels of risk aversion after facing a loss compared with the level of risk aversion after facing a gain. The results did not indicate a significantly different level of risk aversion for losses than for gains. That is, no evidences for loss-aversion preference were found.
} 
then the investors prefers the first asset with the random probabilities over the second asset with the constant probabilities. Since the returns on assets are assumed to be symmetrically distributed, a rational investor who maximizes expected return also minimizes the probability of loss. Thus, if inequality (10) holds a rational investor prefers the asset with the random probability. In other words, he must exhibit ambiguity loving.

Figure 3 provides a graphical representation of inequality (10). This figure assumes two possible normal probability distributions characterized by $\left(\mu_{1}, \sigma_{1}\right)$ and $\left(\mu_{2}, \sigma_{2}\right)$. The y-axes depicts the probability of loss and the x-axes depicts the adjusted reference point (adjusted to the standard normal distribution). Given the random probabilities of loss, the expected probability of loss is $\mathrm{E}[\mathrm{P}(L \mid \mu, \sigma)]$. Assume now a second asset with constant mean, $\mu=\mathrm{E}\left[\mu_{i}\right]=\frac{\mu_{1}+\mu_{2}}{2}$ and constant standard deviation, $\sigma=\mathrm{E}\left[\sigma_{i}\right]=\frac{\sigma_{1}+\sigma_{2}}{2}$. The probability of loss of this asset is $\mathrm{P}(L \mid \mathrm{E}[\mu], \mathrm{E}[\sigma])$. Figure 3 shows a case where the expected probability of loss is smaller than the probability of loss conditional on the expected mean and the expected variance, i.e., $\mathrm{E}[\mathrm{P}(L \mid \mu, \sigma)]<\mathrm{P}(L \mid \mathrm{E}[\mu], \mathrm{E}[\sigma])$.

\section{[ INSERT FIGURE 3 ]}

To check empirically that inequality (10) holds, for each month we compute monthly (i) the expected probability of loss assuming that the mean and variance governing the probability of loss are random and (ii) the probability of loss using the expected mean and expected variance in that month. The average expected probability of loss using (i) is $49.74 \%$, while using (ii) the probability of loss is $50.17 \%$. The difference between (i) and (ii) is negative $(-0.43 \%)$ and significant $(\mathrm{t}=-2.06)$. This result proves that the expected probability of loss when the parameters of the distribution are random, is lower than a constant probability of loss, using the expected parameters. A rational investor, who minimizes the expected probability of loss, prefers (i) over (ii) and therefore by definition he is an ambiguity lover.

Behavioral studies of decision making under ambiguity document that sometimes decision makers exhibit different attitudes toward ambiguity after facing a loss compared with the case where they face a gain (see for example, Bier and Connell (1994), and Chakravarty and Roy (2009a, 2009b)). Different attitudes toward ambiguity can be either different levels of 
ambiguity aversion/seeking or a change in attitude from ambiguity aversion to ambiguity seeking. We tested this hypothesis and found no evidences for different attitudes toward ambiguity. Ambiguity loving was observed for gains and for losses and the degree of ambiguity loving after facing a loss was not significantly different than the level of ambiguity loving after facing a gain.

\section{Conclusions}

The basic tenet in asset pricing is the relationship between risk and return, which has been tested a multitude of times using a variety of models and factors. While this relationship could be tested on the market as a whole using time series data, most of these tests were crosssectional. The results of these tests are mixed at best. In several studies the factor that measures the risk of the asset has a negative coefficient or is non significant while other factors (e.g. liquidity or liquidity risk) turn out to have the desired sign and are significant, which is a puzzle. One possibility is that the missing variable is ambiguity. In this study we introduce for the first time a measure of ambiguity, developed in Izhakian (2011). We use it in conjunction with measures of risk in time series tests.

We claim that excess return on the market as a whole, known as the equity premium, is determined by two orthogonal factors; ambiguity and risk. We measure risk in a variety of ways, e.g., using rate of return variance and implied volatility. Our principle hypothesis is that both of the factors affect the excess return. While, consistent with our asset pricing paradigm of risk aversion, we expect, that the measures of risk will be positively related to the excess return, we have no a-priori view of the effect of ambiguity. The results that we obtain are rather encouraging. The effect of ambiguity is negative and highly significant in all the tests that we employ. This is consistent with several recent studies that show that financial decision makers tend to be ambiguity loving. The effect of risk is generally positive, which is consistent with risk aversion but its significance depends on the risk measure that we use. The best result that we obtain is when we use the unexpected change in volatility as the explanatory variable. Though this is an indirect test of the effect of risk on return, it provides the strongest evidence and is consistent with the results obtained by French, Schwert and Stambaghu (1987). 


\section{References}

Abdellaoui, M., Baillon, A., Placido, L., and Wakker, P, P., 2011, "The Rich Domain of Uncertainty: Source Functions and Their Experimental Implementation," American Economic Review. 101, 2, 695-723.

Amihud, Y. and Hurvich, C. M., 2004, " Predictive Regressions: A Reduced-Bias Estimation Method," Journal of Financial and Quantitative Analysis, 39, 4, 813-841.

Andersen, T. G., Bollerslev, T., Diebold, F. X., and Ebens, H., 2001, "The Distribution of Realized Stock Return Volatility," Journal of Financial Economics, 61, 1, 43-76.

Anderson, E. W., Ghysels, E., and Juergens, J. L., 2009, "The impact of risk and uncertainty on expected returns," Journal of Financial Economics, 94, 2,:233-263.

Bawa, V. S., Brown S., and Klein R., 1979, Estimation Risk and Optimal Portfolio Choice, North Holland, Amsterdam.

Bier, V. M. and Connell, B, L., 1994, “Ambiguity Seeking in Multi-Attribute Decisions: Effects of Optimism and Message Framing,” Journal of Behavioral Decision Making, 7, 169-182.

Brown, D. P., and Gibbons, M. R., 1985 “A Simple Econometric Approach for Utility-Based Asset Pricing Models,” Journal of Finance, 359-381.

Chakravarty, S. and Roy, J., 2009a, "Attitudes towards risk and ambiguity across gains and losses,". Theory and Decision, ISSN 0040-5833

Chakravarty, S. and Roy, J., 2009b, “Recursive Expected Utility and the Separation of Attitudes towards Risk and Ambiguity: An Experimental Study,” Theory and Decision, 66, 199-228.

Chen, Y., Katušcák, P. \& Ozdenoren, E., 2007. "Sealed bid auctions with ambiguity: Theory and experiments," Journal of Economic Theory, 136, 1, 513-535.

Chew, S. H., and Sagi, J. S., 2008, "Small worlds: Modeling attitudes toward sources of uncertainty," Journal of Economic Theory, 139, 1, 1-24.

Chetty, R. 2006. "A New Method of Estimating Risk Aversion," American Economic Review, 96(5): 1821-1834.

Cohen, A., and Einav, L., 2007, "Estimating Risk Preferences from Deductible Choice." American Economic Review, 97, 3, 745-788.

Drechsler, I., 2011, “Uncertainty, Time-Varying Fear, and Asset Prices”, preprint \#1364902 at SSRN.

Ellsberg, D., 1961, "Risk, ambiguity, and the savage axioms," Quarterly Journal of Economics, 75, 4, 643- 669.

Epstein, L., and Schneider, M., 2008, "Ambiguity, information quality, and asset pricing," Journal of Finance, 63, 1, 197-228.

Ergin, H., and Gul, F., 2009, "A theory of subjective compound lotteries," Journal of Economic Theory, 144, 3, 899929.

French, K. R., Schwert and, G. W., and Stambaugh, R. E., 1987, "Expected Stock Returns and Volatility," Journal of Financial Economics, 19, 3-29.

Gilboa, I., 1987, "Expected utility with purely subjective non-additive probabilities," Journal of Mathematical Economics, 16, 1, 65-88.

Gilboa, I., and Schmeidler D., 1989, "Maxmin expected utility with non-unique prior," Journal of Mathematical Economics, 18, 2, 141-153. 
Hansen, L. P. and Sargent, T. J, 2001,. "Robust control and model uncertainty," American Economic Review, 91, 2, $60-66$.

Hayashi, T., and Miao, J., 2011, "Intertemporal substitution and recursive smooth ambiguity preferences," Theoretical Economics, 2011.

Izhakian, Y., 2011, "Shadow Probability Theory for Ambiguity Measurement," preprint \#1937645 at SSRN.

Ivanov, A., 2011, “Attitudes to Ambiguity in One-Shot Normal-Form Games: An Experimental Study,” Games and Economic Behavior, 71, 366-394.

Ju, N. and Miao, J., "Ambiguity, learning, and asset returns," Econometrica, 2011, forthcoming.

Kachelmeier, S. J., and Shehata, M. 1992, "Examining Risk Preferences under High Monetary Incentives: Experimental Evidence from the People’s Republic of China.” American Economic Review, 82, 5, 1120-41.

Kahneman, D., and Tversky, A., 1979, "Prospect theory: An analysis of decision under risk," Econometrica, 47, 2, 263-91.

Klibanoff, P., Marinacci, M. and Mukerji, S. 2005, "A smooth model of decision making under ambiguity," Econometrica, 73, 6, 1849-1892.

Klibanoff, P., Marinacci, M., and Mukerji, S., 2009 "Recursive smooth ambiguity preferences," Journal of Economic Theory, 144, 3, 930-976.

Maffioletti, A., and Santoni, M., 2005, "Do Trade Union Leaders Violate Subjective Expected Utility? Some Insights from Experimental Data”, Theory and Decision, 59, 207-253.

Merton, R. C., 1980, “On Estimating the Expected Return on the Market: An Exploratory Investigation,” Journal of Financial Economics, 8, 323-361.

Nau, R. F., 2006, "Uncertainty aversion with second-order utilities and probabilities," Management Science, 52, $136-145$.

Savage, L. J., 1990, The foundations of statistics reconsidered (1961), 14-20.

Schechter. L. 2007, "Risk aversion and expected-utility theory: A calibration exercise," Journal of Risk and Uncertainty, 35, 1, 67-76.

Schmeidler, D., 1989, "Subjective probability and expected utility without additivity," Econometrica, 57, 3, 571-87.

Segal, U., 1987, "The Ellsberg paradox and risk aversion: An anticipated utility approach," International Economic Review, 28, 1, 175-202.

Tversky, A., and Kahneman, D., 1992, "Advances in prospect theory: Cumulative representation of uncertainty," Journal of Risk and Uncertainty, 5, 4, 297-323.

Uppal, R. and Wang, T., 2003, "Model misspecification and under diversification," Journal of Finance, 58.

Viscusi, W. and Chesson, H., 1999, "Hopes and fears: the conflicting effects of risk ambiguity," Theory and Decision, 47, 153-178.

Von Neumann J. and Morgenstern, O., 1944, Theory of Games and Economic Behavior, University Press.

Wakker, P. P., 2010, Prospect Theory: For Risk and Ambiguity, Cambridge University Press.

Wakker, P. P., Timmermans, D. R.M., and Machielse, I. E., 2007, “The Effects of Statistical Information on Risk and Ambiguity Attitudes, and on Rational Insurance Decisions,” Management Science 53, 1770-1784. 


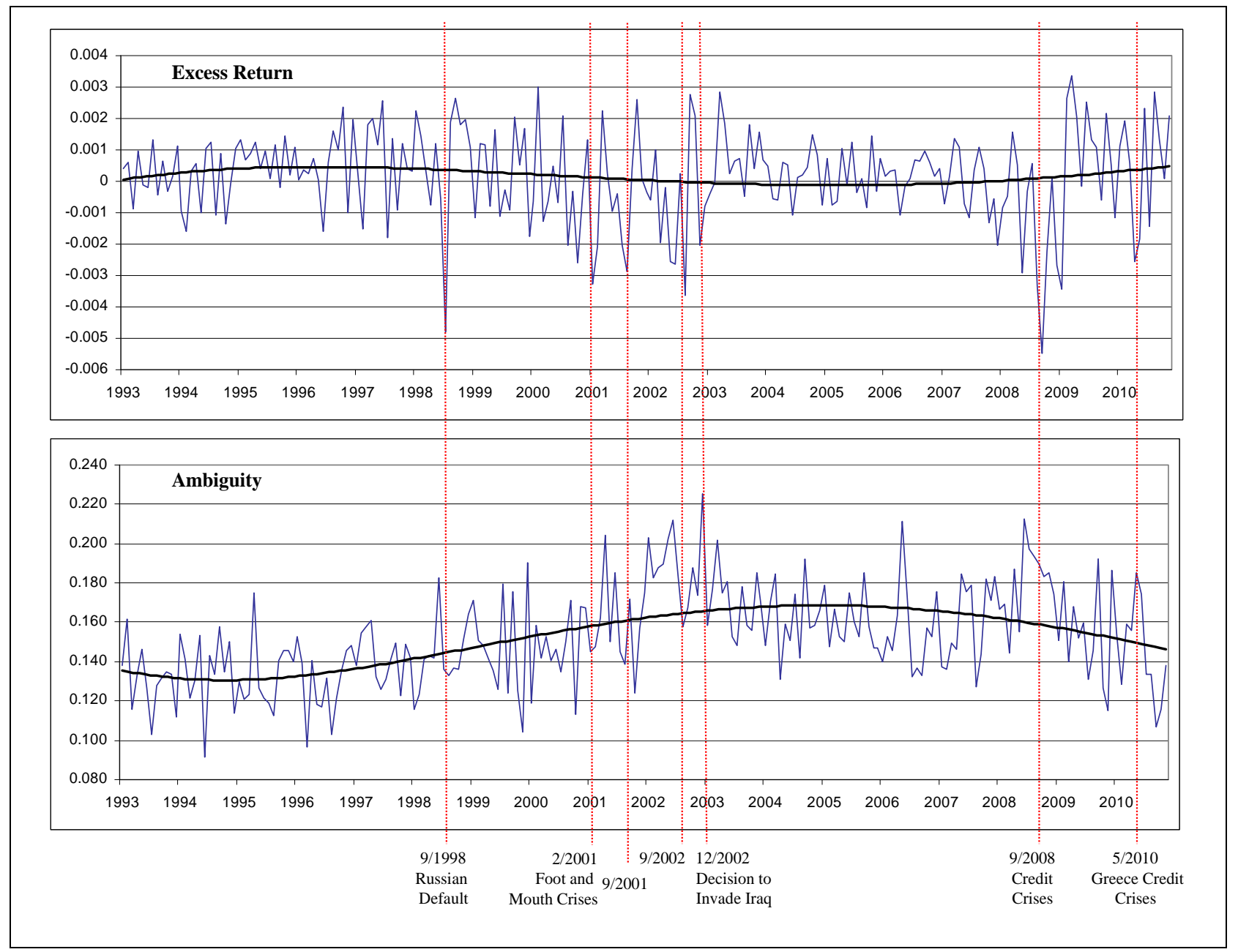

Figure 1: Market excess return and the degree of ambiguity for the period 1993-2010

The upper plot of this figure describes the daily, adjusted for dividend, excess return on the SPY, which serves as a proxy for the market portfolio, between February 1993 and December 2010. The values are the average daily excess return in each month. The lower plot describes the daily level of ambiguity, measured by $\mho$, for each month between February 1993 and December 2010. $\mho$ is computed using 15 minutes rates of return during the month. For each day the probability of loss is computed using the mean and the variance of that day. For each month there are 20-22 probabilities of loss over which the standard deviation is computed to provide the squared degree of ambiguity, $\mho$. The solid smooth lines are created by a polynomial of the $4^{\text {th }}$ degree. The red doted vertical lines designate special events that had a significant impact on the average monthly excess returns. 


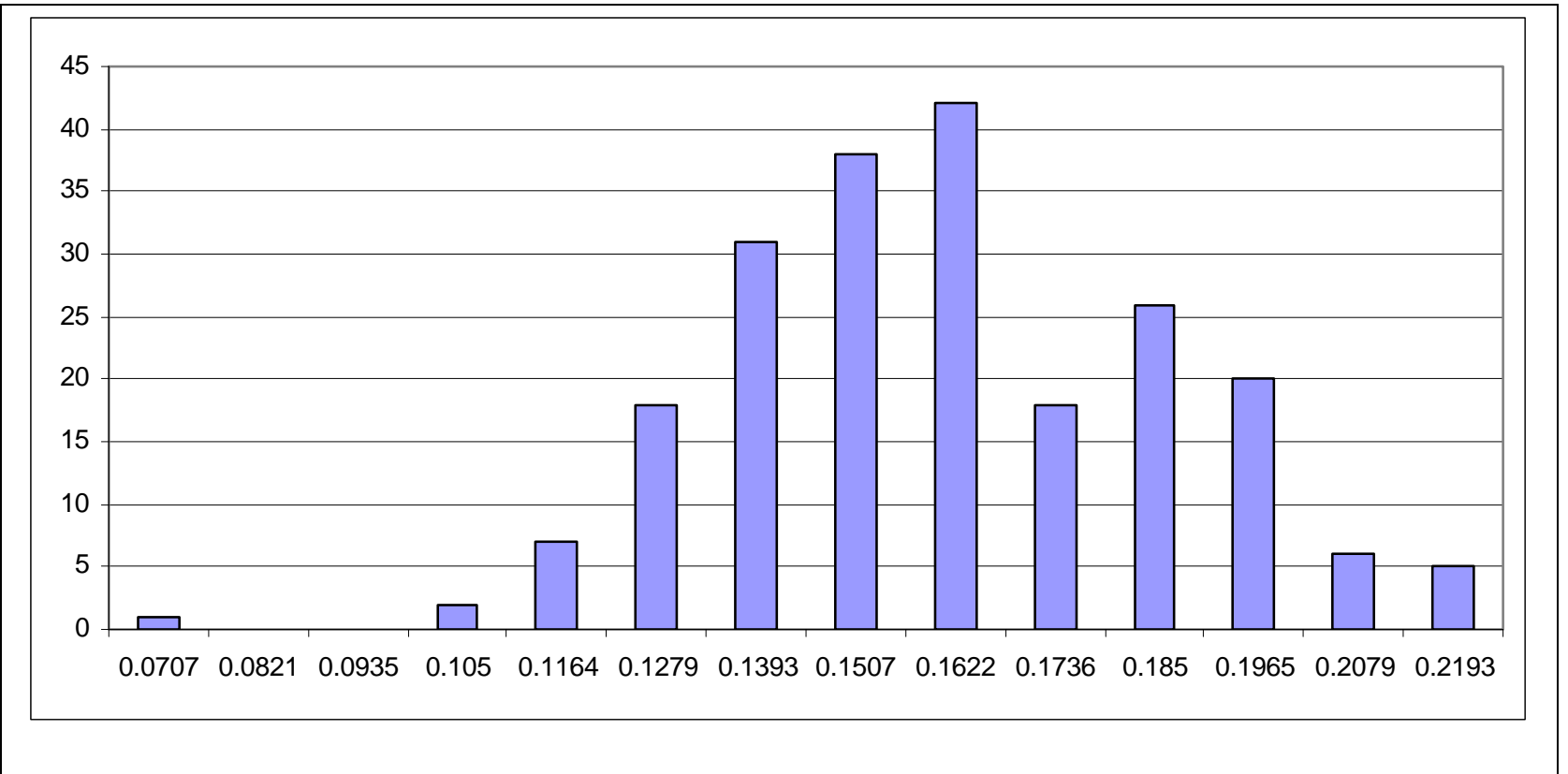

Figure 2: The distribution of ambiguity level.

This figure describes the distribution of the daily level of ambiguity measured by, $\mho$, for the months between February 1993 and December 2010. $\mho$ is computed using 15 minutes rates of return during the month. For each day the probability of loss is computed using the mean and the variance of that day. For each month there are 20-22 probabilities of loss over which the standard deviation is computed to provide the squared degree of ambiguity, $\mho$. Each column depicts the number of observations observed in the range describes on the x-axes. 


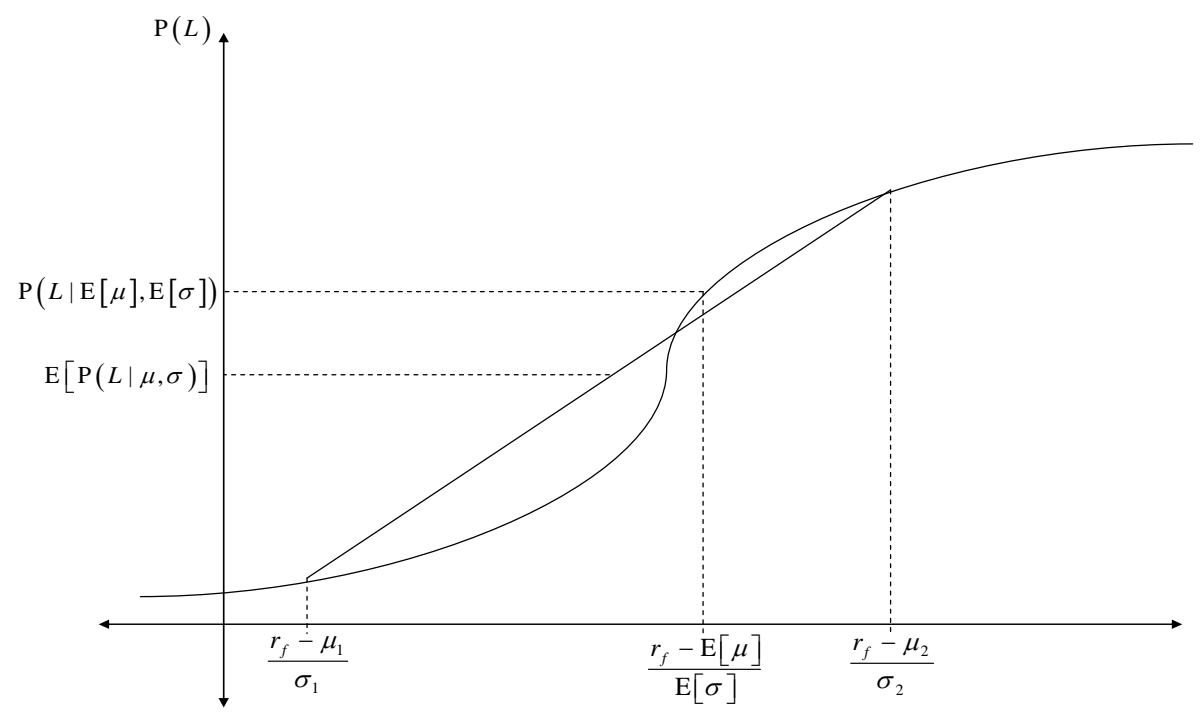

Figure 3: Ambiguity Loving

This figure describes the probability of loss as a function of the threshold differentiating gains form losses, when to probability distribution is normal. The $\mathrm{y}$-axes depicts the probability of loss and the $\mathrm{x}$-axes depicts the value differentiating gains from losses. It assumes two possible normal probability distributions characterized by $\left(\mu_{1}, \sigma_{1}\right)$ and $\left(\mu_{2}, \sigma_{2}\right)$. The expected portability of loss is $\mathrm{E}[\mathrm{P}(L \mid \mu, \sigma)]$. The probability of loss, when the mean and the variance, $(\mathrm{E}[\mu], \mathrm{E}[\sigma])$ are the expected mean and the expected variance, respectively, of $\left(\mu_{1}, \sigma_{1}\right)$ and $\left(\mu_{2}, \sigma_{2}\right)$ is $\mathrm{P}(L \mid \mathrm{E}[\mu], \mathrm{E}[\sigma])$. 


\section{Table I}

\section{Summary Statistics of Regression Variables for the Period 1993-2010}

Panel A reports summary statistics for the entire sample between February 1993 and December 2010. All parameters are normalized to one day. $r_{m}$ is the daily adjusted to dividend return on the SPDR. $r_{f}$ is the daily return on the risk-free asset. MVAR is mean of daily variance. The variance is calculated every day using 15 minutes rates of return (ror) and multiplied by 26 (number of 15 minutes intervals). It is averaged over the number of trading days in a month. VVAR is variance of daily variance. The variance is calculated by using the daily variances during the month. MSKW is mean of daily skweness. The skweness is calculated every day using 15 minute ror, multiplied by 26 and averaged over the month. MKRT is the mean of daily kurtosis. The kurtosis is calculated every day using 15 minute ror multiplied by 26 and averaged over the month. MVIX ${ }^{2}$ is the mean of all daily VIX observation during the month. VVIX ${ }^{2}$ is the variance of all daily VIX observation during the month.

Panel B reports summery statistics for the measure of ambiguity. $\mho^{2}$ is the daily ambiguity level during the month, $\mathrm{E}\left[P_{L}\right]$ is the daily expected probability of loss and $\mho^{2} / \mathrm{E}\left[P_{L}\right]$ - is the normalized measure of ambiguity.

Panel $\mathbf{C}$ reports summery statistics for the volatility indicators. CVAR is the daily variance of the last trading day of the month. CVIX ${ }^{2}$ is the VIX observed in the last trading day of the month. DVIX ${ }^{2}$ is the difference of the observed VIX in the last trading day of the current month and the last trading day of the previous month.

Panel A:

\begin{tabular}{lcccccccc}
\hline & Mean & Variance & Skewness & Kurtosis & Min & Max & Median & N \\
\hline$r_{m}$ & 0.000252 & $2.938 \mathrm{E}-06$ & -0.696084 & 1.018086 & -0.005457 & 0.003368 & 0.000460 & 215 \\
$r_{f}$ & $9.116 \mathrm{E}-05$ & $2.870 \mathrm{E}-09$ & -0.314441 & -1.264361 & 0.000000 & 0.000187 & 0.000103 & 215 \\
$r_{m}-r_{f}$ & 0.000160 & $2.147 \mathrm{E}-06$ & -0.671609 & 0.995231 & -0.005484 & 0.003364 & 0.000328 & 215 \\
$M V A R$ & 0.000121 & $4.142 \mathrm{E}-08$ & 5.777216 & 40.727858 & $1.660 \mathrm{E}-05$ & 0.001787 & $6.925 \mathrm{E}-05$ & 215 \\
$V V A R$ & $8.115 \mathrm{E}-06$ & $8.973 \mathrm{E}-09$ & 14.298609 & 207.352920 & $1.11 \mathrm{E}-09$ & 0.001378 & $3.86 \mathrm{E}-08$ & 215 \\
$M S K W$ & -0.014659 & 0.0241834 & 0.124007 & -0.487596 & -0.365049 & 0.387104 & -0.018444 & 215 \\
$M K R T$ & 0.961168 & 0.5630023 & 1.433304 & 2.859667 & -0.059868 & 4.707871 & 0.762767 & 215 \\
$M V I X^{2}$ & 0.000200 & $4.007 \mathrm{E}-08$ & 3.900617 & 20.981768 & $4.658 \mathrm{E}-05$ & 0.001582 & 0.000158 & 215 \\
$V V I X^{2}$ & $4.654 \mathrm{E}-09$ & $5.459 \mathrm{E}-16$ & 8.935092 & 87.099827 & $8.42 \mathrm{E}-12$ & $2.64 \mathrm{E}-07$ & $4.25 \mathrm{E}-10$ & 215 \\
\hline & & & & Panel B: & & & \\
\hline$\mho^{2}$ & 0.024988 & $6.606 \mathrm{E}-05$ & 0.675753 & 0.240998 & 0.008802 & 0.053262 & 0.023582 & 215 \\
$\mathrm{E}\left[P_{L}\right]$ & 0.497510 & 0.0002281 & 0.050747 & -0.587161 & 0.458340 & 0.538600 & 0.497787 & 215 \\
$\mho^{2} / \mathrm{E}\left[P_{L}\right]$ & 0.050227 & 0.289621 & 13.316224 & -0.410446 & 0.019203 & 0.098890 & 0.047374 & 215 \\
$\mho$ & 0.156041 & 0.0006425 & 0.274433 & -0.221245 & 0.093817 & 0.230787 & 0.153565 & 215 \\
\hline
\end{tabular}




\begin{tabular}{|c|c|c|c|c|c|c|c|c|}
\hline \multicolumn{9}{|c|}{ Panel C: } \\
\hline CVAR & 0.000111 & $3.680 \mathrm{E}-08$ & 8.754059 & 101.518093 & 5.53E-06 & 0.002436 & $6.481 \mathrm{E}-05$ & 215 \\
\hline CVIX ${ }^{2}$ & 0.000198 & 3.319E-08 & 3.209620 & 14.729752 & 4.309E-05 & 0.001423 & 0.000154 & 215 \\
\hline$D V A R$ & $-1.457 \mathrm{E}-07$ & 5.926E-08 & 0.892633 & 61.464498 & -0.002097 & 0.002284 & $-3.00 \mathrm{E}-06$ & 214 \\
\hline$D V I X^{2}$ & 2.631E-07 & $1.232 \mathrm{E}-08$ & 1.735123 & 19.411058 & -0.000578 & 0.000808 & $-1.82 \mathrm{E}-06$ & 214 \\
\hline
\end{tabular}




\section{Table II}

\section{Autocorrelations}

This table reports the autocorrelations of all the different variables, explained and explanatory, which are used in the regressions. The autocorrelations are measured for the period between February 1993 and December 2010. All parameters are normalized to one day. $r_{m}$ is the daily adjusted to dividend return on the SPDR. MVAR is mean of daily variance. The variance is calculated every day using 15 minutes rates of return (ror) and multiplied by 26 (number of 15 minutes intervals). It is averaged over the number of trading days in a month. VVAR is variance of daily variance. The variance is calculated by using the daily variances during the month. MSKW is mean of daily skweness. The skweness is calculated every day using 15 minute ror, multiplied by 26 and averaged over the month. MKRT is the mean of daily kurtosis. The kurtosis is calculated every day using 15 minute ror multiplied by 26 and averaged over the month. $M V I X^{2}$ is the mean of all daily VIX observation during the month. VVIX ${ }^{2}$ is the variance of all daily VIX observation during the month. $\mho^{2}$ is the daily ambiguity level during the month, CVAR is the daily variance of the last trading day of the month. CVIX ${ }^{2}$ is the VIX observed in the last trading day of the month. DVIX ${ }^{2}$ is the difference of the observed VIX in the last trading day of the current month and the last trading day of the previous month.

\begin{tabular}{lcccccc}
\hline & $t-1$ & $t-2$ & $t-3$ & $t-4$ & $t-5$ & $t-6$ \\
\hline$r_{m}-r_{f}$ & 0.0965 & -0.0363 & 0.1213 & 0.0419 & 0.0442 & -0.0481 \\
$\mho^{2}$ & 0.3836 & 0.3930 & 0.3777 & 0.2890 & 0.3499 & 0.3546 \\
$M V A R$ & 0.4399 & 0.2375 & 0.2034 & 0.1837 & 0.0896 & 0.0376 \\
VVAR & -0.0050 & -0.0065 & -0.0064 & -0.0058 & -0.0074 & -0.0075 \\
MVIX ${ }^{2}$ & 0.8498 & 0.6424 & 0.5265 & 0.4601 & 0.3768 & 0.2859 \\
VVIX ${ }^{2}$ & 0.6542 & 0.3492 & 0.0998 & 0.0326 & 0.0226 & -0.0117 \\
MSKW & 0.0262 & 0.1692 & 0.0525 & -0.0388 & 0.1217 & 0.0506 \\
MKRT & 0.6299 & 0.5722 & 0.5702 & 0.5290 & 0.5338 & 0.5944 \\
CVAR & 0.1977 & 0.0834 & 0.0834 & 0.0839 & 0.0840 & 0.0806 \\
CVIX ${ }^{2}$ & 0.8149 & 0.5253 & 0.5253 & 0.4821 & 0.4123 & 0.3192 \\
$D V A R$ & -0.4326 & -0.0033 & -0.0033 & 0.0003 & 0.0021 & 0.0076 \\
$D V I X^{2}$ & 0.0751 & -0.0898 & -0.0898 & 0.0725 & 0.0602 & -0.1295 \\
\hline
\end{tabular}




\section{Table III}

\section{Cross Correlations of Variables}

This table reports the cross-correlations between the different variables, explained and explanatory, which are used in the regressions. The crosscorrelations are measured for the period between February 1993 and December 2010. All parameters are normalized to one day. $r_{m}$ is the daily adjusted to dividend return on the SPDR. MVAR is mean of daily variance. The variance is calculated every day using 15 minutes rates of return (ror) and multiplied by 26 (number of 15 minutes intervals). It is averaged over the number of trading days in a month. VVAR is variance of daily variance. The variance is calculated by using the daily variances during the month. MSKW is mean of daily skweness. The skweness is calculated every day using 15 minute ror, multiplied by 26 and averaged over the month. MKRT is the mean of daily kurtosis. The kurtosis is calculated every day using 15 minute ror multiplied by 26 and averaged over the month. $M V I X^{2}$ is the mean of all daily VIX observation during the month. VVIX ${ }^{2}$ is the variance of all daily VIX observation during the month. $\mho^{2}$ is the daily ambiguity level during the month, E $\left[P_{L}\right]$ is the daily expected probability of loss. CVAR is the daily variance of the last trading day of the month. CVIX ${ }^{2}$ is the VIX observed in the last trading day of the month. DVIX ${ }^{2}$ is the difference of the observed VIX in the last trading day of the current month and the last trading day of the previous month

\begin{tabular}{|c|c|c|c|c|c|c|c|c|c|}
\hline \multicolumn{10}{|c|}{ Panel A: } \\
\hline & $r_{m}-r_{f}$ & $\delta^{2}$ & $\mathrm{E}\left[P_{L}\right]$ & $M V A R$ & VVAR & MSKW & $M K R T$ & $M V I X^{2}$ & $V V I X^{2}$ \\
\hline \multirow{2}{*}{$r_{m}-r_{f}$} & 1.0000 & -0.2294 & -0.7083 & -0.3499 & -0.0513 & -0.0336 & 0.0770 & -0.2853 & -0.3093 \\
\hline & - & $(0.0007)$ & $(<.0001)$ & $(<.0001)$ & $(0.4541)$ & (0.6239) & $(0.2611)$ & $(<.0001)$ & $(<.0001)$ \\
\hline \multirow{2}{*}{$\mho^{2}$} & -0.2294 & 1.0000 & 0.1885 & 0.2042 & 0.0412 & 0.0120 & -0.3183 & 0.2778 & 0.1983 \\
\hline & $(0.0007)$ & - & $(0.0055)$ & $(0.0026)$ & $(0.5478)$ & $(0.8612)$ & $(<.0001)$ & $(<.0001)$ & $(0.0035)$ \\
\hline \multirow{2}{*}{$\mathrm{E}\left[P_{L}\right]$} & -0.7083 & 0.1885 & 1.0000 & 0.1777 & 0.0314 & 0.0432 & -0.1261 & 0.1719 & 0.1025 \\
\hline & $(<.0001)$ & $(0.0055)$ & - & $(0.0090)$ & $(0.6476)$ & $(0.5287)$ & $(0.0651)$ & $(0.0116)$ & $(0.1340)$ \\
\hline \multirow{2}{*}{$M V A R$} & -0.3499 & 0.2042 & 0.1777 & 1.0000 & 0.6132 & 0.1866 & -0.0949 & 0.7339 & 0.6926 \\
\hline & $(<.0001)$ & $(0.0026)$ & $(0.0090)$ & - & $(<.0001)$ & $(0.0061)$ & $(0.1656)$ & $(<.0001)$ & $(<.0001)$ \\
\hline \multirow{2}{*}{ VVAR } & -0.0513 & 0.0412 & 0.0314 & 0.6132 & 1.0000 & 0.1702 & 0.0983 & 0.0629 & 0.0269 \\
\hline & $(0.4541)$ & $(0.5478)$ & $(0.6476)$ & $(<.0001)$ & - & $(0.0124)$ & $(0.1511)$ & $(0.3589)$ & (0.6948) \\
\hline \multirow{2}{*}{$M S K W$} & -0.0336 & 0.0120 & 0.0432 & 0.1866 & 0.1702 & 1.0000 & 0.0498 & 0.1017 & 0.0497 \\
\hline & $(0.6239)$ & $(0.8612)$ & $(0.5287)$ & $(0.0061)$ & $(0.0124)$ & - & $(0.4672)$ & $(0.1374)$ & $(0.4682)$ \\
\hline$M K R T$ & 0.0770 & -0.3183 & -0.1261 & -0.0949 & 0.0983 & 0.0498 & 1.0000 & -0.2851 & -0.0725 \\
\hline
\end{tabular}




\begin{tabular}{ccccccccccc} 
& $(0.2611)$ & $(<.0001)$ & $(0.0651)$ & $(0.1656)$ & $(0.1511)$ & $(0.4672)$ & - & $(<.0001)$ & $(0.2897)$ \\
\multirow{2}{*}{$M V I X^{2}$} & -0.2853 & 0.2778 & 0.1719 & 0.7339 & 0.0629 & 0.1017 & -0.2851 & 1.0000 & 0.7978 \\
& $(<.0001)$ & $(<.0001)$ & $(0.0116)$ & $(<.0001)$ & $(0.3589)$ & $(0.1374)$ & $(<.0001)$ & - & $(<.0001)$ \\
\multirow{2}{*}{$V V I X^{2}$} & -0.3093 & 0.1983 & 0.1025 & 0.6926 & 0.0269 & 0.0497 & -0.0725 & 0.7978 & 1.0000 \\
& $(<.0001)$ & $(0.0035)$ & $(0.1340)$ & $(<.0001)$ & $(0.6948)$ & $(0.4682)$ & $(0.2897)$ & $(<.0001)$ & - \\
\hline
\end{tabular}

\begin{tabular}{|c|c|c|c|c|c|c|}
\hline \multicolumn{7}{|c|}{ Panel B } \\
\hline & $r_{m}-r_{f}$ & $\mho^{2}$ & $C V A R_{t-1}$ & $C V I X_{t-1}$ & DVAR & $D V I X$ \\
\hline \multirow{2}{*}{$r_{m}-r_{f}$} & 1.0000 & -0.2294 & 0.0824 & -0.0091 & -0.3806 & -0.6483 \\
\hline & - & $(0.0007)$ & $(0.2301)$ & $(0.8951)$ & $(<.0001)$ & $(<.0001)$ \\
\hline \multirow{2}{*}{$\mho^{2}$} & -0.2294 & 1.0000 & 0.0125 & 0.2141 & 0.0552 & 0.1007 \\
\hline & $(0.0007)$ & - & $(0.8557)$ & $(0.0016)$ & $(0.4216)$ & $(0.1421)$ \\
\hline \multirow{2}{*}{$C V A R_{t-1}$} & 0.0824 & 0.0125 & 1.0000 & 0.5515 & -0.6332 & -0.1535 \\
\hline & $(0.2301)$ & $(0.8557)$ & - & $(<.0001)$ & $(<.0001)$ & $(0.0248)$ \\
\hline \multirow{2}{*}{ CVIX ${ }_{t-1}^{2}$} & -0.0091 & 0.2141 & 0.5515 & 1.0000 & -0.2182 & -0.3054 \\
\hline & $(0.8951)$ & $(0.0016)$ & $(<.0001)$ & - & $(0.0013)$ & $(<.0001)$ \\
\hline \multirow{2}{*}{$D V A R$} & -0.3806 & 0.0552 & -0.6332 & -0.2182 & 1.0000 & 0.4796 \\
\hline & $(<.0001)$ & $(0.4216)$ & $(<.0001)$ & $(0.0013)$ & - & $(<.0001)$ \\
\hline \multirow{2}{*}{$D V I X^{2}$} & -0.6483 & 0.1007 & -0.1535 & -0.3054 & 0.4796 & 1.0000 \\
\hline & $(<.0001)$ & $(0.1421)$ & $(0.0248)$ & $(<.0001)$ & $(<.0001)$ & - \\
\hline
\end{tabular}




\section{Table IV}

\section{Contemporaneous Regression Tests}

This table presents the contemporaneous regressions, i.e. explaining the market return at time $t$ by the explanatory variables characterized in time $t$. The regressions use data for period between February 1993 and December 2010. All parameters are normalized to one day. The explained variable, $r_{m}$ is the daily adjusted to dividend return on the SPDR. MVAR is mean of daily variance. The variance is calculated every day using 15 minutes rates of return (ror) and multiplied by 26 (number of 15 minutes intervals). It is averaged over the number of trading days in a month. VVAR is variance of daily variance. The variance is calculated by using the daily variances during the month. MKRT is the mean of daily kurtosis. The kurtosis is calculated every day using 15 minute ror multiplied by 26 and averaged over the month. MVIX ${ }^{2}$ is the mean of all daily VIX observation during the month. $V V I X^{2}$ is the variance of all daily VIX observation during the month. $\mho^{2}$ is the daily ambiguity level during the month.

$r_{t}=\alpha+\beta_{1} \mho_{t}^{2}+\beta_{2} M V A R_{t}+\beta_{3} V V A R_{t}+\beta_{4} M V I X_{t}^{2}+\beta_{5} V V I X_{t}^{2}+\beta_{6} M K R T_{t}+\varepsilon_{t}$

\begin{tabular}{|c|c|c|c|c|c|c|c|c|c|}
\hline$\alpha$ & $\mho_{t}^{2}$ & $M V A R_{t}$ & $V V A R_{t}$ & $M V I X_{t}^{2}$ & $V V I X_{t}^{2}$ & $M K R T_{t}$ & $R^{2}$ & Adj $R^{2}$ & $D W$ \\
\hline 0.0012 & -0.0413 & & & & & & 0.0525 & 0.0481 & 1.9875 \\
\hline (3.7636) & $(-3.4285)$ & & & & & & & & \\
\hline 0.0012 & -0.0297 & -2.2773 & & & & & 0.1484 & 0.1403 & 2.0892 \\
\hline (3.9130) & $(-2.5404)$ & $(-4.8742)$ & & & & & & & \\
\hline 0.0012 & -0.0259 & -3.3785 & 3.7486 & & & & 0.1846 & 0.1730 & 2.0546 \\
\hline (4.0144) & $(-2.2439)$ & $(-5.7941)$ & (3.0543) & & & & & & \\
\hline 0.0012 & -0.0294 & & & -1.7589 & & & 0.1058 & 0.0973 & 2.0135 \\
\hline (4.0325) & $(-2.4035)$ & & & $(-3.5442)$ & & & & & \\
\hline 0.0011 & -0.0304 & & & -0.3703 & -14769.4049 & & 0.1259 & 0.1134 & 2.0903 \\
\hline (3.3488) & $(-2.5095)$ & & & $(-0.4625)$ & $(-2.1984)$ & & & & \\
\hline 0.0012 & -0.0411 & & & & & 0.0000 & 0.0526 & 0.0436 & 1.9867 \\
\hline (2.9914) & $(-3.2258)$ & & & & & $(0.0611)$ & & & \\
\hline
\end{tabular}




\begin{tabular}{cccccccccc}
0.0013 & -0.0281 & -3.4286 & 3.8875 & & & -0.0001 & 0.1863 & 0.1708 & 2.0684 \\
$(3.6004)$ & $(-2.3340)$ & $(-5.8327)$ & $(3.1216)$ & & & $(-0.6385)$ & & & \\
0.0011 & -0.0309 & & & -0.4103 & -14510.4128 & 0.0000 & 0.1261 & 0.1094 & 2.0913 \\
$(2.6626)$ & $(-2.4615)$ & & & $(-0.4828)$ & $(-2.0827)$ & $(-0.1423)$ & & & \\
\hline
\end{tabular}




\section{Table V}

\section{Prediction Regression Tests}

This table presents the predictive regressions, i.e. explaining the market return at time $t$ by the explanatory variables characterized in time $t$ - 1 . The regressions use data for period between February 1993 and December 2010. All parameters are normalized to one day. $r_{m}$ is the daily adjusted to dividend return on the SPDR. MVAR is mean of daily variance. The variance is calculated every day using 15 minutes rates of return (ror) and multiplied by 26 (number of 15 minutes intervals). It is averaged over the number of trading days in a month. VVAR is variance of daily variance. The variance is calculated by using the daily variances during the month. MSKW is mean of daily skweness. The skweness is calculated every day using 15 minute ror, multiplied by 26 and averaged over the month. MKRT is the mean of daily kurtosis. The kurtosis is calculated every day using 15 minute ror multiplied by 26 and averaged over the month. $M V I X^{2}$ is the mean of all daily VIX observation during the month. VVIX ${ }^{2}$ is the variance of all daily VIX observation during the month. $\mho^{2}$ is the daily ambiguity level during the month. CVAR is the daily variance of the last trading day of the month. CVIX ${ }^{2}$ is the VIX observed in the last trading day of the month.

\begin{tabular}{|c|c|c|c|c|c|c|c|c|c|c|}
\hline$\alpha$ & $\mho_{t-1}^{2}$ & $M V A R_{t-1}$ & $V V A R_{t-1}$ & $M V I X_{t-1}^{2}$ & $V V I X_{t-1}^{2}$ & $C V A R_{t-1}$ & $C V I X_{t-1}^{2}$ & $R^{2}$ & $\operatorname{Adj} R^{2}$ & $D W$ \\
\hline 0.0013 & -0.0451 & & & & & & & 0.0625 & 0.0581 & 1.9385 \\
\hline$(4.0805)$ & $(-3.7589)$ & & & & & & & & & \\
\hline 0.0013 & -0.0466 & 0.3022 & & & & & & 0.0642 & 0.0553 & 1.9069 \\
\hline (4.0798) & $(-3.8004)$ & $(0.6167)$ & & & & & & & & \\
\hline 0.0013 & -0.0455 & -0.0396 & 1.1629 & & & & & 0.0677 & 0.0543 & 1.9068 \\
\hline$(4.0854)$ & $(-3.6806)$ & $(-0.0635)$ & $(0.8860)$ & & & & & & & \\
\hline 0.0013 & -0.0475 & & & 0.3513 & & & & 0.0646 & 0.0557 & 1.9256 \\
\hline (4.0379) & $(-3.7991)$ & & & (0.6923) & & & & & & \\
\hline 0.0010 & -0.0492 & & & 2.6214 & -24163.5678 & & & 0.1185 & 0.1059 & 2.0224 \\
\hline (3.0691) & $(-4.0437)$ & & & (3.2638) & $(-3.5833)$ & & & & & \\
\hline 0.0012 & -0.0466 & & & & & 0.7915 & & 0.0731 & 0.0644 & 1.8958 \\
\hline (3.9160) & $(-3.8864)$ & & & & & (1.5573) & & & & \\
\hline 0.0013 & -0.0483 & & & & & & 0.5225 & 0.0664 & 0.0575 & 1.9109 \\
\hline (3.9967) & $(-3.8708)$ & & & & & & $(0.9383)$ & & & \\
\hline
\end{tabular}




\section{Table VI}

\section{Regression Tests Using Unexpected Changes}

This table presents the changes regressions, i.e. explaining the market return at time $t$ by the changes in the explanatory variables between time $t$-2and time $t-1$. The regressions use data for period between February 1993 and December 2010. All parameters are normalized to one day. $r_{m}$ is the daily adjusted to dividend return on the SPDR. $\mho^{2}$ is the daily ambiguity level during the month. DVAR is the difference of the observed VAR in the last trading day of the current month and the last trading day of the previous month. DVIX ${ }^{2}$ is the difference of the observed VIX in the last trading day of the current month and the last trading day of the previous month.

\begin{tabular}{ccccccc}
\multicolumn{7}{l}{$r_{m, t}=\alpha+\beta_{1} \mho_{t-1}^{2}+\beta_{2} D V A R_{t}+\beta_{3} D V I X_{t}^{2}+\varepsilon_{t}}$. \\
\hline$\alpha$ & $\mho_{t-1}^{2}$ & $D V A R_{t}$ & $D V I X_{t}^{2}$ & $R^{2}$ & Adj $R^{2}$ & $D W$ \\
\hline 0.0012 & -0.0404 & -2.1990 & & 0.1947 & 0.1870 & 1.9517 \\
$(3.9799)$ & $(-3.6142)$ & $(-5.8852)$ & & & & \\
0.0011 & -0.0379 & & -8.4014 & 0.4642 & 0.4591 & 1.8360 \\
$(4.6313)$ & $(-4.1587)$ & & $(-12.5774)$ & & & \\
0.0002 & & -2.2957 & & 0.1448 & 0.1408 & 1.8091 \\
$(1.7097)$ & & $(-5.9918)$ & & & & \\
0.0002 & & & -8.5763 & 0.4203 & 0.4175 & 1.7145 \\
$(2.1103)$ & & & $(-12.3971)$ & & & \\
\hline
\end{tabular}

